\title{
Visualization of structural changes in cellulosic substrates during enzymatic hydrolysis using multimodal nonlinear microscopy
}

\author{
Ausra Peciulyte $\cdot$ Juris Kiskis • \\ Per Tomas Larsson • Lisbeth Olsson • \\ Annika Enejder
}

Received: 8 December 2015/Accepted: 8 March 2016/Published online: 22 March 2016

(C) The Author(s) 2016. This article is published with open access at Springerlink.com

\begin{abstract}
Enzymatic hydrolysis of cellulose provides a renewable source of monosaccharides for production of variety of biochemicals and biopolymers. Unfortunately, the enzymatic hydrolysis of cellulose is often incomplete, and the reasons are not fully understood. We have monitored enzymatic hydrolysis in terms of molecular density, ordering and autofluorescence of cellulose structures in real time using simultaneous CARS, SHG and MPEF microscopy with the aim of contributing to the understanding and optimization of the enzymatic hydrolysis of cellulose. Three celluloserich substrates with different supramolecular structures,
\end{abstract}

Ausra Peciulyte and Juris Kiskis have contributed equally to this work.

Electronic supplementary material The online version of this article (doi:10.1007/s10570-016-0908-z) contains supplementary material, which is available to authorized users.

A. Peciulyte $\cdot$ L. Olsson $(\bowtie)$

Division of Industrial Biotechnology, Department of Biology and Biological Engineering, Chalmers University of Technology, 41296 Gothenburg, Sweden

e-mail: lisbeth.olsson@chalmers.se

J. Kiskis · A. Enejder

Division of Chemical Biology, Department of Biology and Biological Engineering, Chalmers University of

Technology, 41296 Gothenburg, Sweden

P. T. Larsson

Innventia AB, Drottning Kristinas vag 61, 11428 Stockholm, Sweden pulp fibre, acid-treated pulp fibre and Avicel, were studied at microscopic level. The microscopy studies revealed that before enzymatic hydrolysis Avicel had the greatest carbon-hydrogen density, while pulp fibre and acid-treated fibre had similar density. Monitoring of the substrates during enzymatic hydrolysis revealed the double exponential SHG decay for pulp fibre and acid-treated fibre indicating two phases of the process. Acid-treated fibre was hydrolysed most rapidly and the hydrolysis of pulp fibre was spatially non-uniform leading to fractioning of the particles, while the hydrolysis of Avicel was more than an order of magnitude slower than that of both fibres.

Keywords Cellulose I - Coherent anti-Stokes Raman scattering (CARS) · Second harmonic generation (SHG) - Multiphoton excited fluorescence (MPEF)

\author{
P. T. Larsson \\ Wallenberg Wood Science Center, KTH Royal Institute of \\ Technology, Teknikringen 56, 10044 Stockholm, Sweden \\ L. Olsson \\ Wallenberg Wood Science Center, Chalmers University \\ of Technology, 41296 Gothenburg, Sweden
}




\section{Introduction}

Cellulose is one of the most abundant organic materials on earth, and is found, for example, in forestry and agricultural residues, and in various kinds of waste. Apart from its use in the production of paper and cardboard, cellulose is used in building materials, and in pharmaceuticals, processed food, and cosmetics as a scaffold and texture additive. Interest in further applications of cellulose continues to grow (Klemm et al. 2005). Enzymatic hydrolysis of cellulose provides access to its constituent units, monosaccharides, which can then be converted into a number of products via microbial fermentation (FitzPatrick et al. 2010). Bioethanol is the most mature of the fermentation products, providing renewable transportation fuel (Otero et al. 2007). Unfortunately, the enzymatic hydrolysis of cellulose is often incomplete (Chandra et al. 2007; Igarashi et al. 2011; Zhou et al. 2010), and the arrangement of the supramolecular structures in cellulose is believed to be one of the causes. This is highly dependent on the source of cellulose, and the isolation or pretreatment methods employed. Hence, an understanding of the spatial arrangement of cellulose in different substrates is not only a matter of fundamental scientific interest, but is also of considerable practical importance in developing new materials and improving the efficiency of enzymatic hydrolysis.

Cellulose polymers form a complex, rigid, insoluble structure that is recalcitrant to biological and chemical degradation. The smallest unit of cellulose is a bundle of $\beta$-(1,4)-D-glucan polymer chains with a degree of polymerization varying from 100 to over 15,000 (Zhang and Lynd 2004). In the solid state these polymer chains are packed together, forming fibrils 4-6 nm thick in wood-based cellulose. These fibrils are arranged in fibril aggregates $15-40 \mathrm{~nm}$ thick. In cellulose-rich fibre isolated from e.g. wood, the fibril aggregates form a porous network (Peciulyte et al. 2015).

Various techniques can be used to study cellulose. Spectroscopic techniques provide detailed information about chemical composition and structure of cellulose (Fackler and Schwanninger 2012). The arrangement of the cellulose polymers in bulk samples has been studied with wide and small angle X-ray scattering (Testova et al. 2014) and solid-state crosspolarization magic angle spinning carbon-13 nuclear magnetic resonance (CP/MAS ${ }^{13} \mathrm{C}-\mathrm{NMR}$ ) (Atalla and VanderHart 1999; Foston et al. 2011; Idström et al. 2013; Wickholm et al. 1998). Low-field NMR has been used to study the hydration of cellulose and the relation between water constrained on cellulose surface and saccahrification was observed (Felby et al. 2008; Selig et al. 2013). X-ray photoelectron spectroscopy provides compositional information at both the elemental and functional group levels (Mou et al. 2013). These techniques allow the average properties of materials to be studied, but they lack spatial resolution required to resolve micro- and nano-structures of cellulose.

High resolution imaging techniques such as electron microscopy and scanning probe microscopy are widely used to study cellulose structure (Bubner et al. 2013). These techniques can provide nanoscale information about cellulose structure, but lack chemical specificity, thus revealing mostly topographic details of the specimen. In addition, these techniques often require harsh sample preparation, which may affect the supramolecular structure of the cellulose.

Micro-spectroscopy techniques try to bridge the best of high resolution imaging with high information content of spectroscopic techniques. Time of flight secondary ion mass spectroscopy (Jeremic et al. 2014), infrared absorption (Gierlinger et al. 2008) and Raman scattering techniques (Gierlinger and Schwanninger 2006) allow studying chemical information at submicrometre spatial resolution. Imaging speed of micro-spectroscopy techniques is often limited by the amount of spectral information one wants to retrieve. In order to study dynamic processes, such as enzymatic hydrolysis of cellulose, imaging speed is increased at the expense of spectral information, i.e. single-vibration coherent Raman scattering techniques can achieve video-rate imaging speed, while image with full Raman fingerprint information in each pixel requires acquisition times on the minutes scale (Camp and Cicerone 2015). Other imaging modalities can be implemented together with coherent Raman scattering to form a multimodal nonlinear optical microscope.

Nonlinear microscopy techniques can be used to characterize samples without any sample preparation, as the recorded signals are derived from the intrinsic chemical and physical properties of the sample. In nonlinear microscopy, the signal intensity depends non-linearly on the excitation power. For example, in 
second harmonic generation (SHG) the intensity of the emitted signal scales quadratically with the excitation power. This allows intrinsic 3D optical sectioning, since the signal is effectively generated only in the most intense part of the focused beam. SHG can be used to probe non-centrosymmetric structures, and has been used to image cellulose in cotton (Slepkov et al. 2010; Zimmerley et al. 2010) and rayon fibres (Zimmerley et al. 2010), cellulose derived from the bacterium Acetobacter xylinum (Brackmann et al. 2010; Brown et al. 2003) and the algae Valonia ventricosa (Brown et al. 2003). Multiphoton excited fluorescence (MPEF) can be used to probe the intrinsic fluorescence of the cellulosic substrate, i.e. lignin (Chimenez et al. 2014). Coherent Raman scattering techniques, coherent anti-Stokes Raman scattering (CARS) and stimulated Raman scattering, can probe molecular vibrations, allowing chemically sensitive imaging, for example to image carbohydrates and lignin in plant cell walls. Lignin is generally imaged by probing aromatic ring stretching vibration at $1600 \mathrm{~cm}^{-1}$ (Chen et al. 2010; Ding et al. 2012; Pohling et al. 2014; Saar et al. 2010; Zeng et al. 2010), while polysaccharides can be imaged either by probing $\mathrm{C}-\mathrm{C}$ and $\mathrm{C}-\mathrm{O}$ stretching vibrations at $\sim 1100 \mathrm{~cm}^{-1}$ (Chen et al. 2010; Saar et al. 2010) or C-H stretching vibrations at $\sim 2900 \mathrm{~cm}^{-1}$ (Ding et al. 2012; Pohling et al. 2014; Zimmerley et al. 2010). If full Raman spectrum is recorded, lignin and different types of polysaccharides can be distinguished using spectral fitting (Pohling et al. 2014).

In the present work, we used multimodal nonlinear microscopy, combining SHG, MPEF and CARS to study three different cellulose substrates with different supramolecular properties during enzymatic hydrolysis. Nonlinear microscopy was chosen because: (1) it can be performed in real time, (2) three-dimensional images can be obtained which are necessary for cellulose substrates typically characterized by inhomogeneous morphologies, (3) no sample preparation is required and (4) it provides semi-quantitative information on the chemical composition/density and arrangement. Understanding how such material properties affect hydrolysability can aid in developing cellulosic materials that are more hydrolysable. The substrates used in this study were: (1) never-dried cellulose fibre isolated from softwood by soda pulping, representing 'the best possible case' of commercial pulp specifically designed for high enzymatic reactivity due to the suitable balance between the specific surface area of fibre wall and fibre wall average pore size (referred to as 'the Fibre'); (2) neverdried acid-treated fibre in which the non-crystalline areas had been removed, resulting in a suspension of particles (referred to as 'AT fibre'); and (3) microcrystalline cellulose commercially available as Avicel ${ }^{\circledR} \mathrm{PH}-101$, purchased in powder form, representing a substrate recalcitrant to enzymatic hydrolysis (referred to as 'Avicel'). The samples were chosen to reflect a diversity of cellulose in terms of the size, shape and supramolecular structure. In the previous study differences in the supramolecular structure of the substrates chosen in this study were measured, such as the average pore size (AT fibre $>$ the Fibre $>$ Avicel) and specific surface area (the Fibre $>$ Avicel $>$ AT fibre) (Peciulyte et al. 2015).

\section{Materials and methods}

\section{Cellulosic substrates}

The structural morphologies of the three cellulose-rich substrates used in this study are presented in Table 1 . The Fibre was produced from softwood biomass consisting of an industrially chipped and screened mixture of $40 \%$ Scots pine (Pinus sylvestris) and $60 \%$ Norway spruce (Picea abies) (thickness 2-8 mm). Chips with bark and knots were removed by hand. The chemical composition of the softwood biomass was: $28.3 \%$ acid-insoluble (Klason) lignin, $0.3 \%$ acid-soluble lignin, $41.1 \%$ cellulose, $9.5 \%$ xylan, $17.7 \%$ (galacto)glucomannan, $2.8 \%$ extractives and $0.3 \%$ ash. The major hemicelluloses in softwood are (galacto)glucomannan, galactoglucomannan and arabinoglucuronoxylan (Pettersen 1984). The chips were subjected to pre-hydrolysis followed by alkaline soda cooking, as described elsewhere (Karlström et al. 2014). The Fibres were stored in the never-dried state at $4{ }^{\circ} \mathrm{C}$ until use. AT fibre was produced from the Fibre, according to the procedure described previously by prolonged hydrolysis with hydrochloric acid at elevated temperature (95-100 ${ }^{\circ} \mathrm{C}$ ) for $17 \mathrm{~h}$ (Peciulyte et al. 2014), and stored in excess deionized water in a glass bottle with a lid at room temperature. Avicel ${ }^{\circledR}$ PH-101 was purchased as a powdered microcrystalline cellulose (Fluka BioChemika, Ireland). 
Table 1 The physical characteristics of the cellulosic substrates used in this study

\begin{tabular}{|c|c|c|c|}
\hline Substrate & The fibre & AT fibre & Avicel \\
\hline \multicolumn{4}{|l|}{$\begin{array}{l}\text { Graphical representation (not to } \\
\text { scale) }\end{array}$} \\
\hline Size & $\begin{array}{l}\text { Length: }>2 \mathrm{~mm} \\
\text { Width: } \sim 30 \mu \mathrm{m}\end{array}$ & $\begin{array}{l}\text { Length: } \sim 30 \mu \mathrm{m} \\
\text { Width: } \sim 5 \mu \mathrm{m}\end{array}$ & Diameter: $\sim 50 \mu \mathrm{m}$ \\
\hline Shape & Hollow with closed ends & Rectangular & Irregular \\
\hline Origin & Softwood & Softwood & $\begin{array}{l}\text { Type of wood not stated by } \\
\text { the producer }\end{array}$ \\
\hline Physical state & Never-dried & Never-dried & Dried \\
\hline Treatment & Soda pulping & $\begin{array}{l}\text { Soda pulping and acid } \\
\text { hydrolysis }\end{array}$ & $\begin{array}{l}\text { Acid hydrolysis and spray } \\
\text { drying }\end{array}$ \\
\hline $\begin{array}{l}\text { Conversion yield of cellulose to } \\
\text { glucose after } 2 \text { days of } \\
\text { hydrolysis }^{\mathrm{a}}\end{array}$ & $68 \%$ & $91 \%$ & $31 \%$ \\
\hline
\end{tabular}

${ }^{a}$ Data from Peciulyte et al. (2015)

Analysis of the chemical composition of the cellulosic substrates

The ash content of the biomass and the Fibre was determined according to ISO 1762. The biomass and the Fibre were mixed with acetone prior to analysis of the carbohydrates and lignin to determine the amount of acetone-extractable matter (extractives), according to SCAN-CM 49:03 (2003) standard. The carbohydrates were determined by hydrolysing the samples at $121{ }^{\circ} \mathrm{C}$ in an autoclave with $0.4 \mathrm{M} \mathrm{H}_{2} \mathrm{SO}_{4}$, according to SCAN-CM 71:09 (2009) standard. The solubilized monosaccharides were quantified using high performance anion-exchange chromatography with a Dionex ISC-5000 system coupled to a CarboPac PA1 (250 $\mathrm{mm} \times 4 \mathrm{~mm}$ i.d.) column (Dionex, Sweden) and a pulsed amperometric detector. The samples were hydrolysed with sulphuric acid and then filtered to determine the lignin content. The acid-insoluble (Klason) lignin was determined gravimetrically according to TAPPI T 222 om-02 (2002) method. The acid-soluble lignin was measured by UV spectrophotometry at $205 \mathrm{~nm}$, according to TAPPI UM
250 (1991) method. MilliQ water was used as a blank and for dilution of the hydrolysate in the UV absorption measurements. The acid-soluble fraction was estimated using an absorption coefficient of $110 \mathrm{~L} \mathrm{~g}^{-1} \mathrm{~cm}^{-1}$. The total lignin content was assumed to be the sum of the acid-soluble and acidinsoluble fractions. All samples were analysed in duplicate. The chemical composition of the three substrates used in this study is given in Table 2 .

Sample preparation for the microscopy studies

The cellulosic substrates were imaged prior to enzymatic hydrolysis and followed throughout the process in real time. Different settings were used in the imaging studies, as explained below ("Nonlinear optical microscopy" section). The cellulosic substrates were kept in the closed wells to avoid evaporation of the liquid during the imaging. Sample wells were prepared as follows. Double-sided adhesive spacers (Secure-seal ${ }^{\mathrm{TM}}$ spacer, Invitrogen, thickness $0.12 \mathrm{~mm}$, well diameter $9 \mathrm{~mm}$ ) were covered with a microscope cover glass (Menzel-Gläser, $24 \times 60 \mathrm{~mm}$, thickness $170 \mu \mathrm{m}$ ) to 
Table 2 The chemical composition of the substrates studied in percentage (w/w)

\begin{tabular}{lcll}
\hline Substrate & The fibre & AT fibre & Avicel \\
\hline Acid-insoluble lignin & 0.5 & $<0.5^{\mathrm{a}}$ & n.d. \\
Acid-soluble lignin & 0.5 & $<0.5^{\mathrm{a}}$ & n.d. \\
Extractives & $<0.25$ & n.d. & n.d. \\
Ash content & $<0.1$ & n.d. & n.d. \\
Xylose & 1.5 & 0.8 & 2.2 \\
Mannose & 0.8 & 0.8 & 0.9 \\
Arabinose & $<0.1$ & $<0.1$ & $<0.1$ \\
Galactose & $<0.1$ & $<0.1$ & $<0.1$ \\
Glucose & 96.7 & 98.4 & 96.8 \\
\hline
\end{tabular}

${ }^{a}$ The lignin content of AT fibre was not analysed as the harsh acid hydrolysis used to obtain AT fibres was expected to reduce the lignin levels to amounts below the detection level n.d. not determined

create sample wells. The cellulose samples were applied to these wells and a new cover glass was placed on top. For the measurements on cellulosic substrates prior to enzymatic hydrolysis, the cover glass was coated with $0.01 \%$ poly-L-lysine solution (Sigma, Missouri, USA) and then incubated at $37^{\circ} \mathrm{C}$ overnight. Cellulosic substrates in $6 \mu \mathrm{L}$ deionized water were applied to the wells on the poly-L-lysine-coated cover glass and covered with a second cover glass. For the measurements on cellulosic substrates exposed to enzymatic hydrolysis, a solution of the enzymes was prepared by diluting Celluclast $1.5 \mathrm{~L}(143 \mathrm{mg}$ proteins $\mathrm{mL}^{-1}$ ) and Novozyme 188 ( $\beta$-glucosidase activity 8451 nkat $\mathrm{mL}^{-1}$ ) enzyme mixtures (Novozymes A/S, Bagsvaerd, Denmark) with $50 \mathrm{mM}$ sodium citrate buffer ( $\mathrm{pH} 4.8$ ) 350 and 1400 times, respectively. Novozyme 188 was added to the hydrolysis mixture to compensate for the low $\beta$-glucosidase activity of the Celluclast $1.5 \mathrm{~L}$ mixture. Fresh enzyme solution was prepared for each measurement. A solution with cellulose particles was prepared by mixing cellulose particles with the same buffer to the final concentration of $1 \mathrm{mg} \mathrm{mL}^{-1}$. To study the effects of enzymatic hydrolysis, 1:1 mixture of enzyme solution and cellulose solution was prepared. The enzyme solution and cellulose sample were preheated separately to $40{ }^{\circ} \mathrm{C}$ and then mixed together immediately before imaging. Six $\mu \mathrm{L}$ of the hydrolysis sample (enzyme solution mixed with the cellulose solution) was applied to the well. The aim was to obtain a solution with a few cellulose particles in the final hydrolysis mixture (cellulose sample and enzyme mixture) in order to avoid overlapping of the particles during imaging. Excess enzyme was used to ensure that enzyme concentration was not a limiting factor during enzymatic hydrolysis. The temperature in the incubation chamber (H-301-EC-BL, Okolab) was maintained at $40{ }^{\circ} \mathrm{C}$ during imaging. The cellulosic substrates were not immobilized or mixed during the real-time imaging of hydrolysis. Enzymatic hydrolysis is commonly measured for about $72 \mathrm{~h}$. For the practical reasons in the present study we did not follow enzymatic hydrolysis for more than $9 \mathrm{~h}$. The reaction rate was therefore increased by loading a higher dose of enzymes.

\section{Nonlinear optical microscopy}

Nonlinear optical microscopy measurements were performed using a home-built nonlinear microscope, described in detail elsewhere (Enejder et al. 2010). Briefly, the set-up is based on an inverted laser scanning microscope (Eclipse TE-2000-E, Nikon) and a picosecond pulsed laser source consisting of a pump laser (1064 nm, Nd:Vanadate, 7 ps, $76 \mathrm{MHz}$, Picotrain, HighQ Lasers) and an optical parametric oscillator (OPO, 700-980 nm; Emerald, APE). Laser beams from both units were spatially and temporally overlapped and guided into the entrance of the microscope laser scanner. CARS and SHG signals were measured in the transmission direction, while MPEF was measured in the epidirection. A high numerical aperture objective (Plan Fluor $40 \times, 1.3$ NA, Nikon) was used to obtain efficient excitation of nonlinear signals. The excitation power at the sample was approximately $40 \mathrm{~mW}$ for each of the excitation beams.

CARS experiments were conducted at a Raman shift of $2880 \mathrm{~cm}^{-1}$, which is characteristic of the $\mathrm{C}-\mathrm{H}$ stretching vibration of the $\mathrm{CH}_{2}$ group in cellulose (Zimmerley et al. 2010). The $1064 \mathrm{~nm}$ output was used as the Stokes beam and the output of the OPO was tuned to $814 \mathrm{~nm}$ and used as the pump beam, resulting in CARS emission at $659 \mathrm{~nm}$. The CARS signal was filtered with two short-pass filters (FF01-790/SP-25, Semrock) and a single bandpass filter (FF01-661/2025 , Semrock) and detected by a single-photon-counting photomultiplier tube (PMC-100-20, Becker and Hickl). Both laser sources contributed to multiphoton excited red autofluorescence (detected in the wavelength region of 580-637 nm), which was filtered with two short-pass filters (FF01-790/SP-25, Semrock) and 
a single bandpass filter (FF01-609/57-25, Semrock), and detected by a single-photon-counting photomultiplier tube (HPM-100-40, Becker and Hickl). In the SHG measurements, emission was recorded at $407 \mathrm{~nm}$, which corresponds to the second harmonic generated by the beam from the OPO tuned to $814 \mathrm{~nm}$. The SHG signal was filtered with two short-pass filters (FF01-790/SP-25, Semrock) and a single bandpass filter (FF01-405/10-25, Semrock), and detected by a single-photon-counting photomultiplier tube (HPM100-40, Becker \& Hickl).

When imaging cellulosic substrates before enzymatic hydrolysis the voxel size was $100 \mathrm{~nm}$ in the $\mathrm{xy}-$ direction and $500 \mathrm{~nm}$ in the z-direction, the pixel dwell time was $61.4 \mu \mathrm{s}$, and the image size (in pixels) was adjusted according to the size of each particle. When monitoring the effects of enzymatic hydrolysis on the substrates, the image size was fixed at $512 \times 512$ pixels and $40 \mathrm{z}$-slices, with a voxel size of $586 \mathrm{~nm}$ in the xydirection and $1000 \mathrm{~nm}$ in the z-direction, and the pixel dwell time was $18 \mu \mathrm{s}$, resulting in a total 3D frame size of $150 \times 150 \times 40 \mu \mathrm{m}$ and a time of $3.6 \mathrm{~min}$.

Image analysis

The images were analysed with Image (Image J 1.50a, NIH) (Schneider et al. 2012). Poisson noise, inherent to single-photon-counting detection, was removed using the ImageJ plugin PureDenoise, based on wavelet denoising of Poisson noise corrupted images (Luisier et al. 2010). The decays of nonlinear signals (SHG, MPEF, CARS) were calculated as follows. (1) The signal from the particle was separated from the background using a single-level threshold; (2) the threshold value was subtracted from the signal above the threshold; (3) the total intensity over the particle was calculated by integrating the threshold-subtracted signal; and (4) the total intensity at different time points was normalized to the total intensity at the beginning of the enzymatic hydrolysis experiment.

\section{Results and discussion}

Nonlinear microscopy of the cellulosic substrates

The cellulosic substrates were imaged prior to hydrolysis (three particles of each substrate, one particle of each substrate shown in Fig. 1). The data for different particles is qualitatively consistent, i.e. signal strength from one substrate is stronger than from another substrate, while there were quantitative differences between particles of the same substrate (i.e. size of the particle, absolute strength of the signal). Two sets of images were acquired for each substrate: (1) with laser polarization parallel to the long axis of the particles (parallel polarization) and (2) with laser polarization perpendicular to the long axis of the particles (perpendicular polarization). All nonlinear signals showed a dependence on the laser polarization, which reflects the highly ordered structure of the cellulosic substrates. Data in Fig. 1 is plotted on a logarithmic scale as the signals span wide range of intensities (almost 4 orders of magnitude).

SHG imaging with parallel polarization of the laser revealed narrow linear structures of high intensity along the long axis of the Fibre and AT fibre particles, while in Avicel the regions of high SHG intensity were distributed less regularly, but still mainly followed the long axis of the particle (Fig. 1a, xy view, // symbol). When imaged with perpendicular polarization of the laser, the SHG intensity was much lower for all substrates, usually by more than 1 order of magnitude (Fig. 1a, xy view, $\perp$ symbol).

SHG can arise only in non-centrosymmetric structures. Glucan chains lack inversion symmetry and have previously been shown to give rise to SHG in starch (Brackmann et al. 2011; Slepkov et al. 2010) and cellulosic substrates of different origin (Brackmann et al. 2010; Brown et al. 2003; Glas et al. 2015; Pohling et al. 2014; Slepkov et al. 2010; Zimmerley et al. 2010). In order to interpret the SHG images of cellulosic substrates, the coherent nature of the SHG signal and the supramolecular structure of cellulose must be taken into account. In incoherent imaging modalities, such as fluorescence, the total detected intensity is proportional to the number and brightness of the individual emitters, i.e. the total detected intensity, $I_{\text {total }}$, is the sum of the emission intensities, $I_{n}$, of each individual emitter, $n: I_{\text {total }} \sim \sum I_{n}$. In coherent imaging modalities, the total detected electric field is proportional to the coherent sum of the electric field intensities of the individual emitters, i.e. the total detected intensity, $I_{\text {total }}$, is proportional to the square of the sum of the electric fields, $\boldsymbol{E}_{\boldsymbol{n}}=E_{n} e^{i_{\varphi_{n}}}$ (where $\varphi_{n}$ is the phase of the field at the detector) 
Fig. 1 Multimodal nonlinear optical microscopy of the three cellulosic substrates. Second-harmonic generation (SHG) (a), multiphoton excited fluorescence (MPEF) (b) and coherent anti-Stokes Raman scattering at $2880 \mathrm{~cm}^{-1}$ (C-H CARS) (c) contrast images of the Fibre, AT fibre and Avicel. Imaging was performed with laser polarization parallel (upper rows, // symbol) and perpendicular (lower rows, $\perp$ symbol) to the long axis of the particles. Polarization of the laser is indicated by the white arrows on the right side of the images. Images represent a single optical slice through the particle in the xy- and zy-directions. Note that the colour scale is logarithmic and differs between the panels. Voxel size: $100 \mathrm{~nm}$ in the xydirection and $500 \mathrm{~nm}$ in the z-direction. Pixel dwell time: $61.4 \mu \mathrm{s}$. Scale bar $10 \mu \mathrm{m}$
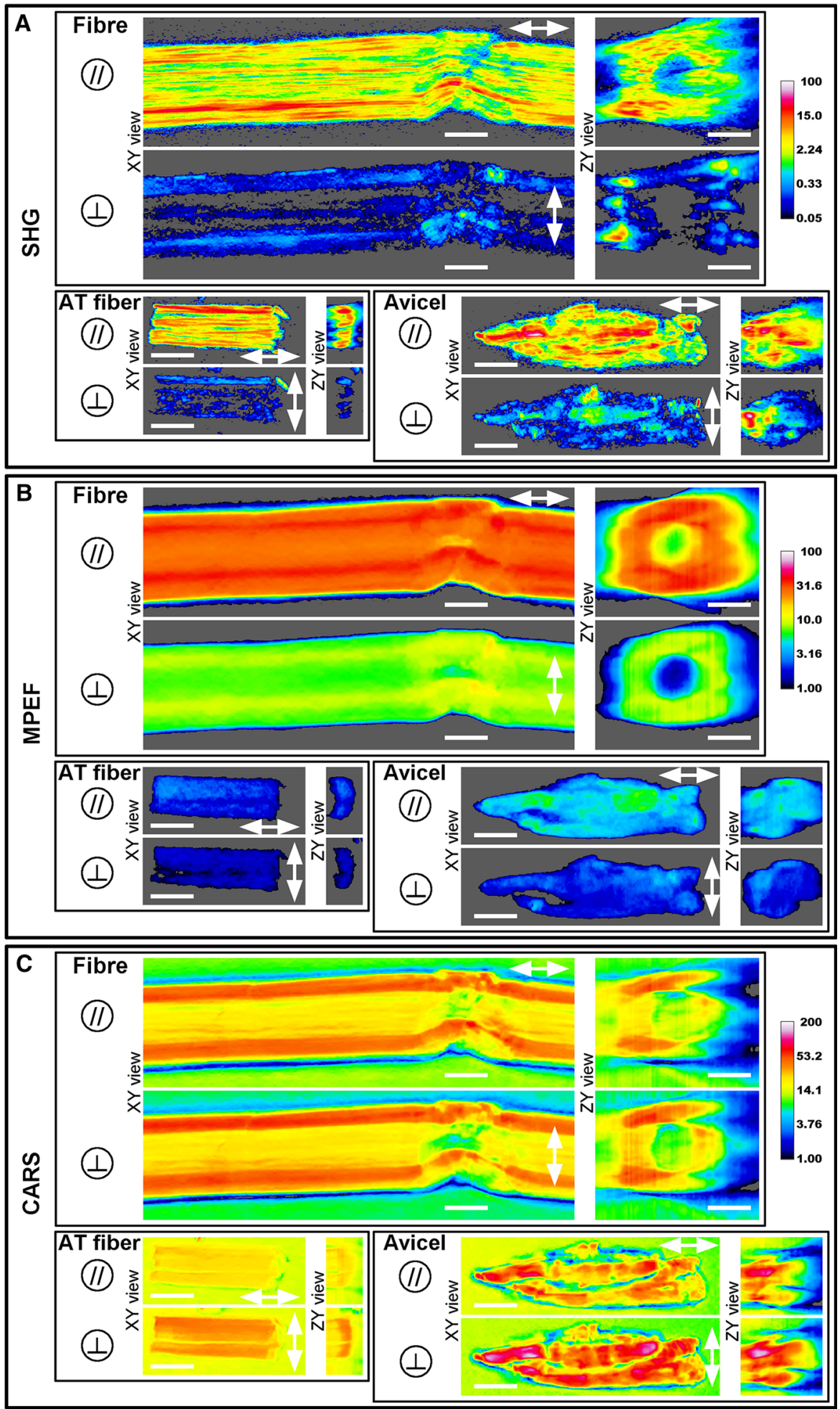
emitted by each individual emitter $n: I_{\text {total }} \sim\left(\sum E_{n}\right.$ $\left.e^{i_{\varphi_{n}}}\right)^{2}$. The coherent nature of the SHG signal has two important implications: (1) the total signal is proportional to the square of the number of emitters, and (2) the total signal is modulated by the relative phase of the electric fields generated by the individual emitters. The latter means that the SHG signal is sensitive to the relative spatial orientation/order of individual glucan chains. Strong SHG signals are emitted from $\beta-(1,4)-$ D-glucan chains arranged parallel as in cellulose I (i.e. cotton fibres), in contrast to weak or no SHG signals from $\beta$ - $(1,4)$-D-glucan chains arranged anti-parallel as in cellulose II (i.e. rayon fibres) (Zimmerley et al. 2010). The SHG signal has been shown to be strongest when the polarization of the excitation beam is parallel to the dipoles of the molecules, which, in the case of cellulose, is along the $\beta$-(1,4)-D-glucan polymer (Brown et al. 2003).

Cellulosic substrates used in our study were derived from wood cells. Plant cell walls are composed of cellulose layers where fibril aggregates are oriented at specific angles, called microfibril angles, with respect to the axis of the cell. A plant cell has a primary wall and a secondary wall, the latter having three layers (S1, S2 and S3). The thickness of the S2 layer is much greater than that of the S1 and S3 layers. This means that for measurements that involve the whole thickness of a single cell wall, the results will be dominated by the angle of the S2 layer, which has a smaller microfibril angle with respect to the axis of the cell than the other layers (Barnett and Bonham 2004). It is therefore probable that the image in Fig. 1a represents the S2 layer in the cell wall. It has been suggested previously that the SHG signal in cellulose-rich samples originates from fibril aggregates (Brown et al. 2003). In native plant cell walls, fibril aggregates are imbedded in the hemicellulose and lignin matrix. In the substrates studied here, the hemicellulose and lignin had been removed to a large extent. Fibre wall regions initially rich in hemicellulose and lignin are less dense after cellulose isolation due to submicrometre voids, and such regions appear as areas of lower intensity in the SHG images. The Fibre and AT fibre were kept in the never-dried state, and there was, therefore, no irreversible collapse of cellulose structures due to hornification, which is a plausible explanation of the repeating regions of strong and weak SHG signal (Fig. 1a). Drying and subsequent re- wetting of cellulose is known to induce persistent, possibly irreversible, changes in its supramolecular structure, responsible for the phenomenon known as hornification, which reduces its water-binding capacity (Chunilall et al. 2010), in turn reducing its accessibility by enzymes. Avicel had undergone harsh acid hydrolysis and subsequent drying (Reier 2000). It has been shown that drying causes aggregation of the fibrils into large aggregates (Chunilall et al. 2010), which could explain the irregular distribution of strong SHG signal in Avicel particle (Fig. 1a).

MPEF images of all three cellulosic substrates showed a relatively uniform distribution of intensities throughout the particle volume (Fig. 1b). Similarly to the SHG images, MPEF emission excited with perpendicular laser polarization was weaker than that with parallel laser polarization. Polarization sensitivity of MPEF indicates that absorption dipoles of the fluorophore molecules are preferentially oriented relative to the polarization of the excitation beam. The Fibre showed the highest MPEF intensities, roughly 5 times stronger than Avicel and 10 times stronger than AT fibre (for both excitation polarizations). There may be several sources of autofluorescence in wood fibres. Lignin is a highly fluorescent component of plant-derived cellulosic substrates. The high MPEF intensity of the Fibre is in line with the Fibre having higher amounts of lignin than Avicel and AT fibre (Table 2), although there may also be contributions from other autofluorescent species. Uniform intensity of the autofluorescence throughout the particle volume and higher emission intensity with parallel laser polarization were also observed in a recent study of bleached fibre derived from sugarcane bagasse (Chimenez et al. 2014). Chimenez et al. (2014) suggested that the uniform distribution of MPEF intensity throughout the particle could be explained by the dispersion of lignin and other fluorophores in the particle during pretreatment, while no explanation for the fluorescence polarization sensitivity has been provided. Polarization sensitivity of the fluorescence indicates that substantial numbers of the fluorophores are preferentially aligned relative to the cellulose fibrils in all three substrates studied. Without knowledge about the particular fluorophores leading to the fluorescence polarization sensitivity it is difficult to draw more detailed conclusions regarding the origin of polarization sensitivity. 


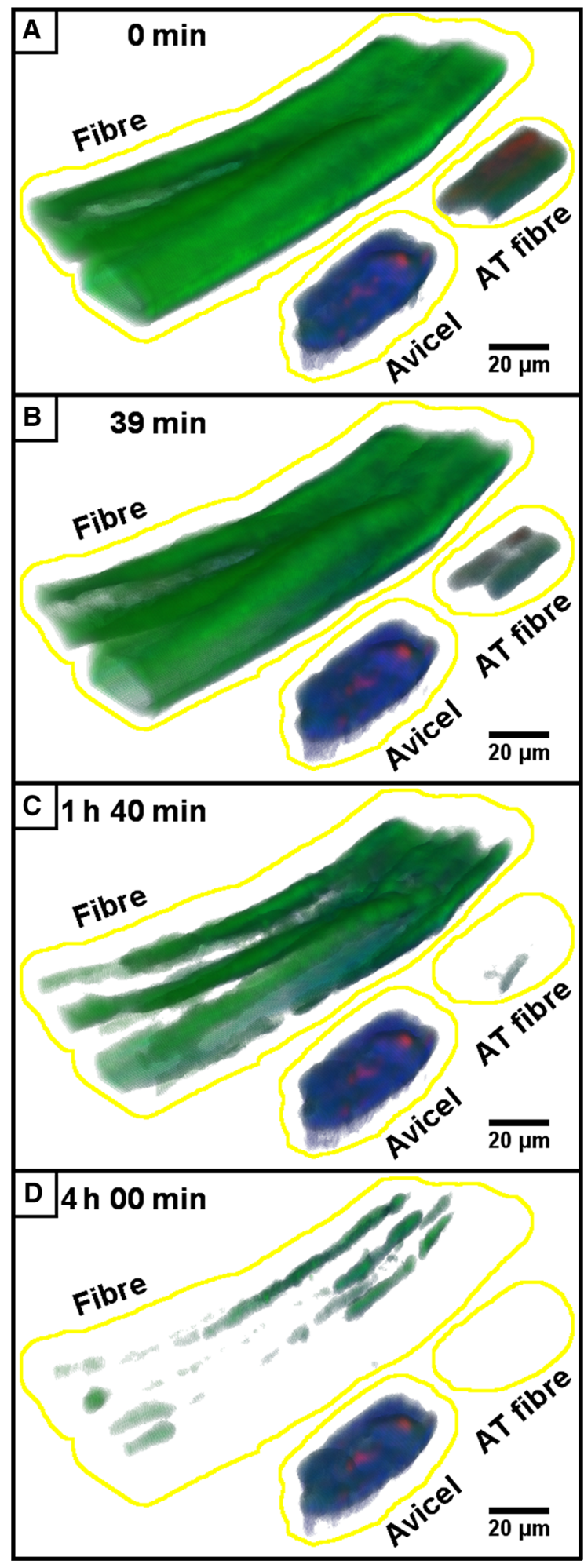

4Fig. 2 Volume rendering of nonlinear microscopy of the Fibre, AT fibre and Avicel during enzymatic hydrolysis. Substrates at the beginning of the experiment (a), after $39 \mathrm{~min}$ (b), $1 \mathrm{~h}$ $40 \mathrm{~min}(\mathbf{c})$ and $4 \mathrm{~h}(\mathbf{d})$ of hydrolysis. Imaging modalities are represented in different colours: SHG in red, MPEF in green and CARS in blue. The yellow lines are to guide the eye. Voxel size: $586 \mathrm{~nm}$ in the xy-direction and $1000 \mathrm{~nm}$ in the z-direction. Pixel dwell time: $18 \mu \mathrm{s}$, total 3D frame time: $3.6 \mathrm{~min}$. (Color figure online)

The CARS intensity of all particles was higher for perpendicular than parallel polarization of the laser. The Avicel particles showed the highest CARS intensity; roughly twice stronger than of the Fibre and 3 times stronger than of AT fibre for both excitation polarizations (Fig. 1c). The ratio of the signal intensities with excitation using perpendicular and parallel polarization was quite different for the three substrates: approximately 2 for Avicel, 1.5 for AT fibre and 1.1 for the Fibre. Quantitative comparison of CARS signals is complicated because CARS intensity depends on the density of $\mathrm{CH}_{2}$ groups in a nonlinear fashion; furthermore the background signal from water was relatively strong. Nevertheless, since these factors affect all CARS images in the similar way, CARS intensities can be compared qualitatively, i.e. higher CARS signal reflect higher density of $\mathrm{CH}_{2}$ groups, which should be proportional to the amount of $\beta$ - $(1,4)$-D-glucan polymers. Thus we can deduce that the cellulose in Avicel particles was packed more densely than in the Fibre or AT fibre; the latter being the least dense. Avicel undergoes drying, which is known to cause the irreversible collapse of the structures, and thus a higher density, which was reflected by a larger density of $\mathrm{CH}_{2}$ groups. AT fibre was produced by prolonged acid hydrolysis of the Fibre, which is known to remove non-crystalline domains of cellulose and induce polymer scission. This results in the transformation of cellulose-rich fibres into a sol of cellulose micro- and nano-particles with a large surface area accessible, for example, to enzymatic hydrolysis due to removal of the fibre wall. Reduced density of AT fibre due to prolonged acid hydrolysis results in low CARS signal. The interpretation of relative differences in CARS intensity resulting from two orthogonal excitation polarizations is complicated due to possible interference from the vibrationally nonresonant electronic contribution with 


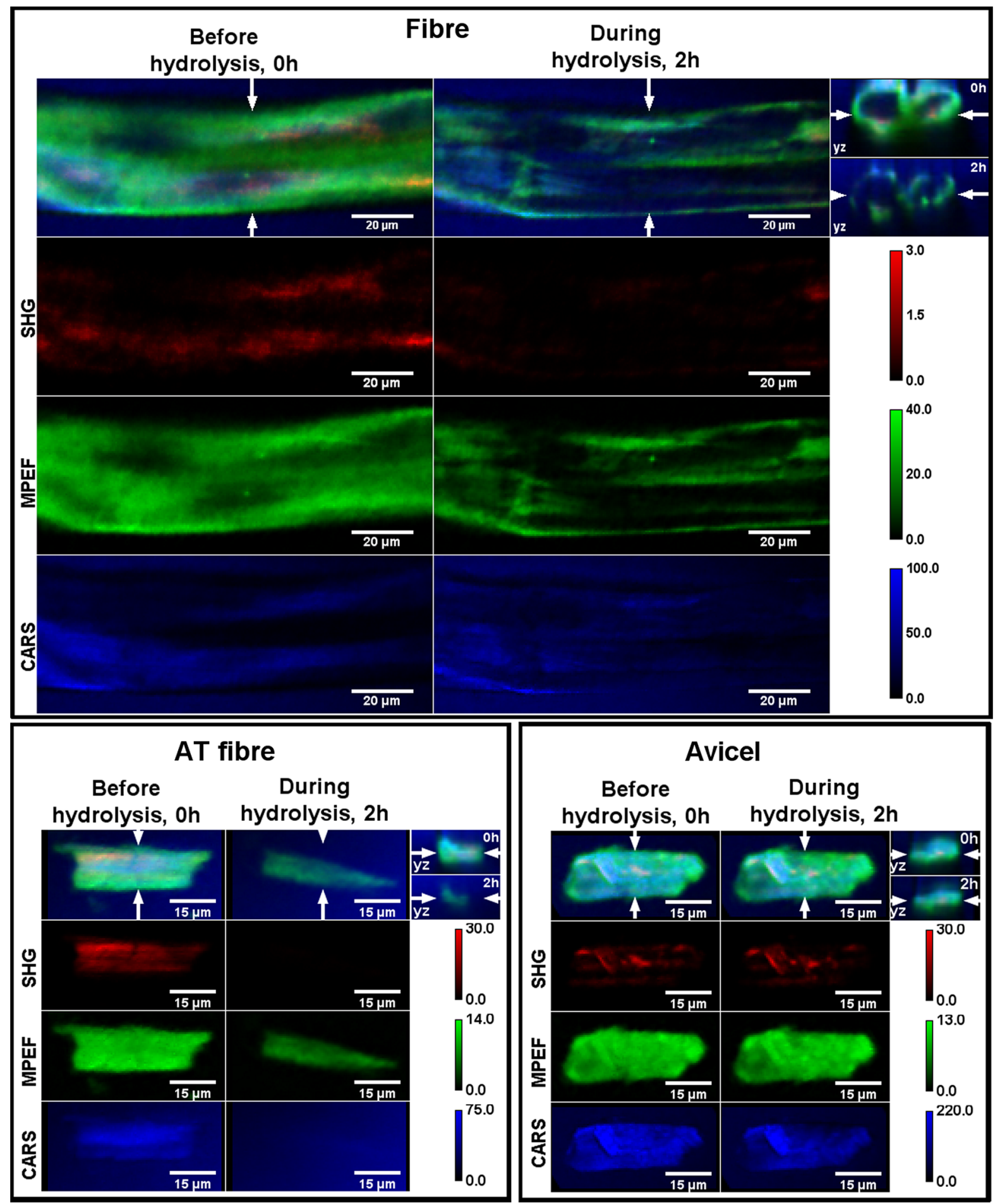

different polarization properties than the $\mathrm{C}-\mathrm{H}$ CARS signals measured (Zimmerley et al. 2010), especially for relatively thin AT fibre particles, leading to a reduction in the difference between the signals at the two polarizations. Higher CARS intensities were expected with perpendicular laser polarization, since 
4 Fig. 3 Nonlinear microscopy of the Fibre, AT fibre and Avicel before $(0 \mathrm{~h})$ and during hydrolysis $(2 \mathrm{~h})$. The upper rows show the overlay image of the three nonlinear contrast mechanisms used: SHG in red, MPEF in green and C-H CARS in blue; the three rows below show single channel images of the corresponding contrast mechanism. It was not possible to control the orientation of the particles during hydrolysis, thus, in order to avoid orientation-related signal variation, each image represents the sum of the images obtained at two orthogonal excitation laser polarizations. Images represent a single optical slice through the particle in the xy- and zy-directions, and the white arrows show the position of the cross-sections. Note that the colour scale differs between the panels. Voxel size: $586 \mathrm{~nm}$ in the xy-direction and $1000 \mathrm{~nm}$ in the $\mathrm{z}$-direction. Pixel dwell time: $18 \mu \mathrm{s}$, total 3D frame time: $3.6 \mathrm{~min}$. (Color figure online)

the $\mathrm{C}-\mathrm{H}$ bonds are perpendicular to the axis of $\beta-(1,4)$ D-glucan polymers, which should be mostly aligned or have a small microfibril angle along the long axis of the particles, if we assume that the image represents the S2 layer of the secondary plant cell wall (Barnett and Bonham 2004). This is supported by the SHG measurements of the same particles (Fig. 1a), and previous studies showing that emission peaks in CARS and SHG signals are orthogonal to each other in terms of excitation polarization for cotton fibre (Slepkov et al. 2010; Zimmerley et al. 2010) and starch (Slepkov et al. 2010).

Structural changes in the cellulosic substrates during enzymatic hydrolysis

Imaging of enzymatic hydrolysis in real-time (Fig. 2, time-lapse is available as Supplementary Video 1) revealed that the Fibre and AT fibre were more hydrolysable than Avicel (one particle of each substrate was imaged). The Fibre and AT fibre particles showed a significant decrease in volume after less than $1 \mathrm{~h}$ of enzymatic hydrolysis (Fig. 2a vs. b).

In contrast, no significant changes were seen in the volume of the Avicel particles after $4 \mathrm{~h}$ of enzymatic hydrolysis (Fig. 2a vs. d). This is in agreement with the conversion yields of cellulose to glucose reported previously (Peciulyte et al. 2015). Although the Fibre exhibited the highest MPEF signal (Fig. 1,2), which was attributed to the presence of lignin (Chimenez et al. 2014), hydrolysis of the Fibre was quite rapid. Lignin is known to have a negative impact on enzymatic hydrolysis (Zeng et al. 2014), which may in part account for the slower hydrolysis of the Fibre than AT fibre. The Fibre was observed to split into elongated parts during hydrolysis. The effect of enzymatic hydrolysis was not evenly distributed along the axis of the Fibre as some parts of the Fibre were initially hydrolysed without significant change in the length of the Fibre while other parts remained intact. This indicates that there were parts within the Fibre of varying hydrolysability (Fig. 2). It has been shown in a previous study that during enzymatic hydrolysis pulp fibres preferably scission at dislocations, which have a typical distance of about $20 \mu \mathrm{m}$ along the fibre (Thygesen et al. 2011). It has been concluded from some studies that fibres derived from various sources were more hydrolysable at dislocations (BuschleDiller et al. 1994; Clarke et al. 2011; Gourlay et al. 2015; Gurnagul et al. 1992). We did not observe faster hydrolysis perpendicular to the long axis of the Fibre, which would have indicated the presence of dislocations. We were only able to observe part of the Fibre (hundreds of micrometres compared to several millimetres length of the intact fibre), and we cannot exclude that fractioning at dislocations took place outside the field-of-view. Another possible explanation of why we did not observe fragmentation perpendicular to the axis of the Fibre could be that the reactants were not mixed. It has been shown that enzymatic fragmentation of fibres is more apparent when the reaction is agitated, consequently enhancing the mechanically mediated fragmentation of the fibres (Arantes et al. 2014).

No segmentation of AT fibre particle was observed during enzymatic hydrolysis, but the particle shrank without significant change in the length. It has previously been suggested that when a fibre reaches a certain particle size during hydrolysis, then deconstruction proceeds predominantly by swelling and peeling or erosion, rather than by further fibre fragmentation (Arantes et al. 2014). This finding is in agreement with the results of our study, where no fragmentation was observed during the enzymatic hydrolysis of AT fibre.

Some details of the effect of enzymatic hydrolysis on the substrates shown in Fig. 2 were even more evident in optical cross-sections of the particles (Fig. 3). In the volume rendering of the particles in Fig. 2 the inner parts of the particles are not visible, while the optical cross-sections shown in Fig. 3 reveal the changes in the chosen section of the particle. Due to the fast scanning during the real-time imaging of hydrolysis, the background signal in CARS 
microscopy (Fig. 3, blue channel) is large, making it difficult to distinguish faint structures in the end of hydrolysis. The particles moved or decreased in size during enzymatic hydrolysis, and optical cross-sections in Fig. 3 before $(0 \mathrm{~h})$ and during $(2 \mathrm{~h})$ hydrolysis were therefore manually selected to represent the same areas of the particles as closely as possible.

Spatially non-uniform hydrolysis of the Fibre was apparent when comparing images before and during hydrolysis (Fig. 3a). The SHG signal decreased substantially during $2 \mathrm{~h}$ of hydrolysis, while certain small areas of high intensity were still present (Fig. 3a, red channel). The MPEF signal decreased over large parts of the particle, leaving areas of almost unchanged intensity elongated in the direction of the long axis of the particle (Fig. 3a, green channel), which was seen as fractioning in the volume rendering of the particle (Fig. 2c, d Fibre). Particle changes observed in the CARS signal were similar to the changes observed in the MPEF signal, but weak structures were obscured by the background (Fig. 3a, blue channel). During the hydrolysis of AT fibre, the SHG signal disappeared faster than the MPEF signal, and after $2 \mathrm{~h}$ of hydrolysis the SHG image was empty (Fig. 3b, red channel), while the MPEF signal showed a slightly rotated particle of lower intensity and reduced size (Fig. 3b, green channel). After $2 \mathrm{~h}$ of hydrolysis no resonant CARS signal could be distinguished (Fig. 3b, blue channel). In contrast, no obvious changes were seen in any of the images of Avicel substrate.

The uniform decrease in the SHG signal after $2 \mathrm{~h}$ of hydrolysis of the Fibre and AT fibre suggests that disruption of the ordered structures occurred over the whole cellulose particle. The SHG signal indicated that all three substrates contain well-ordered, parallelaligned glucan chains to a large extent. Ordered structures might indicate fewer obstacles for enzymatic action, while roughness of the cellulose surface could lead to the congestion of enzymes, as discussed by Igarashi et al. (2011). AT fibre was shown to be ordered, and was also the least dense (the Fibre had a slightly higher density) (Figs. 1c, 3a, c), which is likely to provide good access for enzymes. This is in well agreement of the fast hydrolysis of AT fibre observed in our study. In contrast, the presence of strong SHG and CARS signals in Avicel (Figs. 1, 3) suggests that apart from the ordered structure of Avicel, the particle was also the most dense of the three substrates studied, which may have limited the access of enzymes to the $\beta$ - $(1,4)$-D-glucan polymers, confirmed by slow enzymatic hydrolysis observed in the SHG and CARS images.

The overall decay of the nonlinear signals due to enzymatic hydrolysis is shown in Fig. 4. The normalized intensity was calculated by integrating the threshold-subtracted signal intensity over the volume of the particle and dividing by the integrated intensity at the beginning of the experiment. The noise in the curves is mainly due to the movement of the particles. All three nonlinear signals decayed fastest during the hydrolysis of AT fibre (Fig. 4b), followed by the Fibre (Fig. 4a) and then Avicel (Fig. 4c). The SHG decay profiles for the Fibre and AT fibre have two components: an initial slower decay rate (during the first $\sim 12$ min of the experiment for AT fibre and $\sim 50 \mathrm{~min}$ for the Fibre), followed by a faster decay rate during the rest of hydrolysis (Fig. 4a, b inserts). Initial decay rate of SHG for the Fibre and AT fibre is similar to the corresponding decay rate of MPEF but became faster towards the end of hydrolysis. The SHG decay profile of Avicel exhibited a slow and constant decay rate (Fig. 4c insert). In contrast, the decay of the MPEF signal for Avicel was much slower than the corresponding SHG decay. The CARS decay profile for AT fibre does not resemble either the SHG or MPEF profile (Fig. 4b). As discussed above in the case of Fig. 3, the background signal interfered with the CARS signal during enzymatic hydrolysis experiments, which manifests as a faster decay of the CARS signal at lower CARS intensities. AT fibre exhibited the lowest CARS intensity, thus the CARS decay of AT fibre was most affected, seen as faster decay of CARS signals compared to decay of SHG and MPEF signals. Despite interference from the background, we can conclude that the CARS decay profile for the Fibre showed a decay rate similar to that of MPEF, while the CARS decay profile for Avicel was similar to that of SHG, but not MPEF.

The low signal decay rate observed in Avicel (Fig. 4c) can be attributed to low accessibility of the enzymes due to the high ordering and density of the material (Fig. 1a, c). The highest rate of signal decay observed in AT fibre (Fig. 4b) can be attributed to the high accessibility by enzymes due to the low density of the material (Fig. 1c). The initially low decay rate of the SHG signal during hydrolysis of the Fibre and AT fibre indicated that the outer layers of the particles 

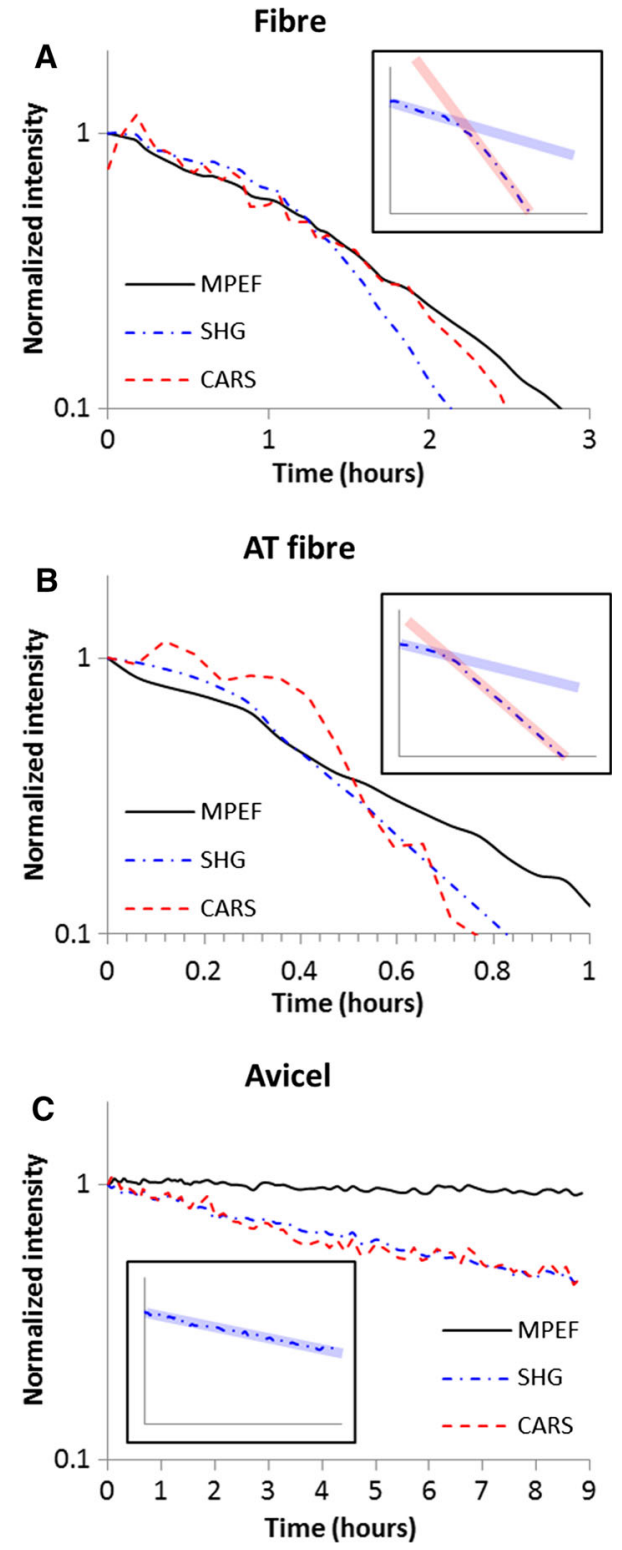

Fig. 4 Decay of nonlinear signals during enzymatic hydrolysis of the Fibre (a), AT fibre (b) and Avicel (c). The graphs show normalized intensities of the SHG, MPEF and CARS signals integrated over the whole particle, representing the average decay of the signal over the volume of the particles shown in Figs. 2 and 3. Note the logarithmic scale of the vertical axis

were less prone to hydrolysis. One possible explanation of this is that at the beginning of enzymatic hydrolysis of the Fibre and AT fibre the fibre wall morphology may limit enzymatic accessibility. Since AT fibre was derived from the Fibre with most of the fibre wall morphology removed, the initial phase of hydrolysis was shorter, and the decay rate faster, than that of the Fibre.

It has been reported previously that the SHG signal decreased smoothly during the enzymatic hydrolysis of Valonia cellulose, but no explicit explanation of the physical/chemical origin of the signal decrease was provided (Brown et al. 2003). In a study on ionic liquid treatment of cellulose, the decrease in SHG signal during cellulose particle swelling has been explained as being the consequence of cellulose amorphization and a decrease in cellulose crystallinity (Glas et al. 2015). The fact that the SHG decay was faster than the MPEF decay for all substrates in our study may indicate that disordering of cellulose takes place before the fluorophores are released from disordered and/or disrupted material. SHG intensity can decrease not only as a result of disordering of cellulose, but also due to the disruption of material. The $\mathrm{C}-\mathrm{H}$ CARS signal reflects the density of the material, and the reduction in the CARS signal thus indicates disruption of the material and the release of soluble components into the buffer solution. Since the decay rates of the SHG and CARS signals differed only slightly during the hydrolysis of the Fibre and AT fibre, the degree of disordering and/or the period of time during which disordering preceded disruption of the material should be relatively small. Taken together, the evidence indicates that the processes of cellulose disordering, the release of fluorophores and the disruption of the material took place closely in time.

In contrast to the Fibre and AT fibre, the decrease in the SHG signal during the hydrolysis of Avicel was much more prominent than the decrease in the MPEF signal, which remained almost constant. The fact that the decrease in the CARS signal closely followed the decrease in the SHG signal indicates that disordering of the cellulose was closely followed by disruption of material. The fact that the MPEF signal from Avicel did not decrease, despite substantial disordering and disruption of cellulose, indicates that the fluorophores in Avicel were distributed in the areas of the particle highly recalcitrant to hydrolysis.

\section{Conclusions}

We have characterized three cellulose-rich substrates, the Fibre, AT fibre and Avicel, using simultaneous SHG, MPEF and CARS nonlinear microscopy. Using 
SHG microscopy we probed the ordering of glucan chains averaged over a sub-micrometre volume of the focused laser beam. The SHG pattern, i.e. the regularity of low- and high-intensity regions, allowed us to distinguish the never-dried Fibre and AT fibre (more regular) from Avicel (less regular), possibly reflecting the collapse of the macromolecular structures in Avicel during drying and rewetting. The distribution of intrinsic fluorophores was imaged using MPEF microscopy. A uniform distribution of fluorophores was found in the particles of all three substrates, possibly as a result of bleaching treatment during the production of the particles. The density of cellulose was imaged using CARS microscopy sensitive to the $\mathrm{CH}_{2}$ group. The Fibre and AT fibre had similar densities, while Avicel was found to be the most dense, supporting the evidence from SHG of collapsed macromolecular structures in Avicel.

Real-time nonlinear microscopy allowed us to study changes in the supramolecular structure of these cellulose-rich substrates. Volume renderings of the particles showed the effects of hydrolysis on the wholeparticle level. AT fibre was hydrolysed most rapidly without any significant change in the particle length, the Fibre showed spatially non-uniform hydrolysis leading to the fractioning of the particle, and hydrolysis of Avicel was more than an order of magnitude slower than for the other two substrates, leaving the shape of the particle almost unchanged. The time resolution of our imaging experiments was sufficient to distinguish two phases of structural changes occurring during hydrolysis of the Fibre and the AT fibre. Our interpretation was that during the initial phase, hydrolysis of the fibre wall components proceeds more slowly due to limited enzymatic accessibility, followed by a more rapid hydrolysis phase occurring when the enzyme accessibility restricting fibre wall morphology had been broken down.

Using nonlinear optical microscopy we were able to monitor the changes in cellulose supramolecular structure during enzymatic hydrolysis. The structural differences observed between the three cellulose-rich substrates were related to their hydrolysability. The possibility of relating structural properties of cellulose with micrometre resolution to the enzymatic hydrolysability at single-particle level illustrates the potential of nonlinear microscopy to add to our knowledge of the complex supramolecular structures of cellulose. We believe that such knowledge will help in developing new materials with better hydrolysability and improving the efficiency of enzymatic hydrolysis.

Acknowledgments This work was funded by the Swedish Research Council under the Programme for Strategic Energy Research under Grant Agreement No. 621-2010-3788 (LO and AP), the Knut and Alice Wallenberg Foundation through the Wallenberg Wood Science Center (LO), the People Programme (Marie Curie Actions) of the European Union's Seventh Framework Programme FP7/2007-2013/under REA Grant Agreement No. 607842 (JK) and RiSE Research Institutes of Sweden AB (PTL). Authors are grateful to Henning Hagman for discussions and help with experiments during the initial phase of the study.

Open Access This article is distributed under the terms of the Creative Commons Attribution 4.0 International License (http:// creativecommons.org/licenses/by/4.0/), which permits unrestricted use, distribution, and reproduction in any medium, provided you give appropriate credit to the original author(s) and the source, provide a link to the Creative Commons license, and indicate if changes were made.

\section{References}

Arantes V, Gourlay K, Saddler JN (2014) The enzymatic hydrolysis of pretreated pulp fibers predominantly involves "peeling/erosion" modes of action. Biotechnol Biofuels 7:1-11

Atalla R, VanderHart DL (1999) The role of solid state 13C NMR spectroscopy in studies of the nature of native celluloses. Solid State Nucl Magn Reson 15:1-19

Barnett J, Bonham VA (2004) Cellulose microfibril angle in the cell wall of wood fibres. Biol Rev 79:461-472

Brackmann C, Bodin A, Akeson M, Gatenholm P, Enejder A (2010) Visualization of the cellulose biosynthesis and cell integration into cellulose scaffolds. Biomacromolecules 11:542-548

Brackmann C, Bengtsson A, Alminger ML, Svanberg U, Enejder A (2011) Visualization of beta-carotene and starch granules in plant cells using CARS and SHG microscopy. J Raman Spectrosc 42:586-592

Brown RM Jr, Millard AC, Campagnola PJ (2003) Macromolecular structure of cellulose studied by second-harmonic generation imaging microscopy. Opt Lett 28: 2207-2209

Bubner P, Plank H, Nidetzky B (2013) Visualizing cellulase activity. Biotechnol Bioeng 110:1529-1549

Buschle-Diller G, Zeronian S, Pan N, Yoon M (1994) Enzymatic hydrolysis of cotton, linen, ramie, and viscose rayon fabrics. Text Res J 64:270-279

Camp CH Jr, Cicerone MT (2015) Chemically sensitive bioimaging with coherent Raman scattering. Nat Photonics 9:295-305

Chandra RP, Bura R, Mabee WE, Berlin A, Pan X, Saddler JN (2007) Substrate pretreatment: the key to effective 
enzymatic hydrolysis of lignocellulosics? Adv Biochem Eng Biotechnol 108:67-93. doi:10.1007/10_2007_064

Chen B-C, Sung J, Lim S-H (2010) Chemical imaging with frequency modulation coherent anti-Stokes Raman scattering microscopy at the vibrational fingerprint region. J Phys Chem B 114:16871-16880

Chimenez TA, Gehlen MH, Marabezi K, Curvelo AAS (2014) Characterization of sugarcane bagasse by autofluorescence microscopy. Cellulose 21:653-664

Chunilall V, Bush T, Larsson PT, Iversen T, Kindness A (2010) A CP/MAS ${ }^{13} \mathrm{C}$-NMR study of cellulose fibril aggregation in eucalyptus dissolving pulps during drying and the correlation between aggregate dimensions and chemical reactivity. Holzforschung 64:693-698

Clarke K, Li X, Li K (2011) The mechanism of fiber cutting during enzymatic hydrolysis of wood biomass. Biomass Bioenergy 35:3943-3950

Ding S-Y, Liu Y-S, Zeng Y, Himmel ME, Baker JO, Bayer EA (2012) How does plant cell wall nanoscale architecture correlate with enzymatic digestibility? Science 338:1055-1060

Enejder A, Brackmann C, Svedberg F (2010) Coherent antiStokes Raman scattering microscopy of cellular lipid storage. IEEE J Sel Top Quantum Electron 16:506-515

Fackler K, Schwanninger M (2012) How spectroscopy and microspectroscopy of degraded wood contribute to understand fungal wood decay. Appl Microbiol Biotechnol 96:587-599

Felby C, Thygesen LG, Kristensen JB, Jørgensen H, Elder T (2008) Cellulose-water interactions during enzymatic hydrolysis as studied by time domain NMR. Cellulose 15:703-710

FitzPatrick M, Champagne P, Cunningham MF, Whitney RA (2010) A biorefinery processing perspective: treatment of lignocellulosic materials for the production of value-added products. Bioresour Technol 101:8915-8922

Foston $M$ et al (2011) Chemical, ultrastructural and supramolecular analysis of tension wood in Populus tremula $\times$ alba as a model substrate for reduced recalcitrance. Energy Environ Sci 4:4962-4971

Gierlinger N, Schwanninger M (2006) Chemical imaging of poplar wood cell walls by confocal Raman microscopy. Plant Physiol 140:1246-1254

Gierlinger N, Goswami L, Schmidt M, Burgert I, Coutand C, Rogge T, Schwanninger M (2008) In situ FT-IR microscopic study on enzymatic treatment of poplar wood crosssections. Biomacromolecules 9:2194-2201

Glas D, Paesen R, Depuydt D, Binnemans K, Ameloot M, De Vos DE, Ameloot R (2015) Cellulose amorphization by swelling in ionic liquid/water mixtures: a combined macroscopic and second-harmonic microscopy study. ChemSusChem 8:82-86

Gourlay K, Hu J, Arantes V, Penttilä M, Saddler JN (2015) The use of carbohydrate binding modules (CBMs) to monitor changes in fragmentation and cellulose fiber surface morphology during cellulase- and swollenin-induced deconstruction of lignocellulosic substrates. J Biol Chem 290:2938-2945

Gurnagul N, Page DH, Paice MG (1992) The effect of cellulose degradation on the strength of wood pulp fibres. Nord Pulp Pap Res J 7:152-154
Idström A, Brelid H, Nydén M, Nordstierna L (2013) CP/MAS ${ }^{13} \mathrm{C}$ NMR study of pulp hornification using nanocrystalline cellulose as a model system. Carbohydr Polym 92:881-884

Igarashi K et al (2011) Traffic jams reduce hydrolytic efficiency of cellulase on cellulose surface. Science 333:1279-1282

Jeremic D, Goacher RE, Yan R, Karunakaran C, Master ER (2014) Direct and up-close views of plant cell walls show a leading role for lignin-modifying enzymes on ensuing xylanases. Biotechnol Biofuels 7:496

Karlström K, Sjögren B, Vorwerg W, Volkert B (2014) Sulphurfree cooking for value added cellulose. Cell Chem Technol 48:781-786

Klemm D, Heublein B, Fink HP, Bohn A (2005) Cellulose: fascinating biopolymer and sustainable raw material. Angew Chem Int Ed 44:3358-3393

Luisier F, Vonesch C, Blu T, Unser M (2010) Fast interscale wavelet denoising of Poisson-corrupted images. Signal Process 90:415-427

Mou H-Y, Orblin E, Kruus K, Fardim P (2013) Topochemical pretreatment of wood biomass to enhance enzymatic hydrolysis of polysaccharides to sugars. Bioresour Technol 142:540-545

Otero J, Panagiotou G, Olsson L (2007) Fueling industrial biotechnology growth with bioethanol. In: Olsson L (ed) Biofuels, vol 108., Advances in biochemical engineering/ biotechnologySpringer, Berlin, pp 1-40

Peciulyte A, Anasontzis GE, Karlström K, Larsson PT, Olsson L (2014) Morphology and enzyme production of Trichoderma reesei Rut $\mathrm{C}-30$ are affected by the physical and structural characteristics of cellulosic substrates. Fungal Genet Biol 72:64-72

Peciulyte A, Karlström K, Larsson PT, Olsson L (2015) Impact of the supramolecular structure of cellulose on the efficiency of enzymatic hydrolysis. Biotechnol Biofuels 8:56

Pettersen RC (1984) The chemical-composition of wood. Adv Chem Ser 207:57-126

Pohling C, Brackmann C, Duarte A, Buckup T, Enejder A, Motzkus M (2014) Chemical imaging of lignocellulosic biomass by CARS microscopy. J Biophotonics 7:126-134

Reier EG (2000) Avicel PH microcrystalline cellulose, NF, Ph Eur. JP, BP FMC Corp Sec 11:1-27

Saar BG, Zeng Y, Freudiger CW, Liu YS, Himmel ME, Xie XS, Ding SY (2010) Label-free, real-time monitoring of biomass processing with stimulated Raman scattering microscopy. Angew Chem Int Ed 49:5476-5479

SCAN-CM (2003) SCAN-CM standard 49:03. Content of acetone-soluble matter. Scandinavian pulp, paper and board testing committee

SCAN-CM (2009) SCAN-CM standard 71:09. Carbohydrate composition. Scandinavian pulp, paper and board testing committee

Schneider CA, Rasband WS, Eliceiri KW (2012) NIH Image to ImageJ: 25 years of image analysis. Nat Methods 9:671-675

Selig MJ, Thygesen LG, Johnson DK, Himmel ME, Felby C, Mittal A (2013) Hydration and saccharification of cellulose I $\beta$, II and IIII at increasing dry solids loadings. Biotechnol Lett 35:1599-1607

Slepkov AD, Ridsdale A, Pegoraro AF, Moffatt DJ, Stolow A (2010) Multimodal CARS microscopy of structured carbohydrate biopolymers. Biomed Opt Express 1:1347-1357 
TAPPI (1991) TAPPI useful method UM 250: acid-soluble lignin in wood and pulp

TAPPI (2002) TAPPI test method T 222 om-02: acid-insoluble lignin in wood and pulp

Testova L, Borrega M, Tolonen LK, Penttilä PA, Serimaa R, Larsson PT, Sixta H (2014) Dissolving-grade birch pulps produced under various prehydrolysis intensities: quality, structure and applications. Cellulose 21:2007-2021

Thygesen LG, Hidayat BJ, Johansen KS, Felby C (2011) Role of supramolecular cellulose structures in enzymatic hydrolysis of plant cell walls. J Ind Microbiol Biotechnol 38:975-983

Wickholm K, Larsson PT, Iversen T (1998) Assignment of noncrystalline forms in cellulose I by $\mathrm{CP} / \mathrm{MAS}{ }^{13} \mathrm{C} \mathrm{NMR}$ spectroscopy. Carbohydr Res 312:123-129

Zeng Y et al (2010) Imaging lignin-downregulated alfalfa using coherent anti-Stokes Raman scattering microscopy. BioEnergy Res 3:272-277
Zeng Y, Zhao S, Yang S, Ding S-Y (2014) Lignin plays a negative role in the biochemical process for producing lignocellulosic biofuels. Curr Opin Biotechnol 27:38-45

Zhang Y-HP, Lynd LR (2004) Toward an aggregated understanding of enzymatic hydrolysis of cellulose: noncomplexed cellulase systems. Biotechnol Bioeng 88:797-824

Zhou W, Xu Y, Schüttler HB (2010) Cellulose hydrolysis in evolving substrate morphologies III: time-scale analysis. Biotechnol Bioeng 107:224-234

Zimmerley M, Younger R, Valenton T, Oertel DC, Ward JL, Potma EO (2010) Molecular orientation in dry and hydrated cellulose fibers: a coherent anti-stokes Raman scattering microscopy study. J Phys Chem B 114:1020010208 